\title{
Innovations en région, développement en métropole ?
}

\author{
Richard Shearmur \\ INRS-Urbanisation, Culture et Soci t
}

\section{Introduction}

Depuis une quinzaine d'années, une littérature abondante a mis en avant et développé le concept de « milieu innovateur ». Ce concept est intimement lié à ceux de l'économie du savoir et de la nouvelle économie, car on y souligne les effets du savoir, du réseautage et de la circulation de l'information sur la croissance économique. Dans ce contexte propice, le concept de milieu innovateur ainsi que l'ensemble des concepts antérieurs ou dérivés (système régional d'innovation, district industriel, grappe industrielle) est devenu une pièce maîtresse de la pensée et de la pratique en matière de développement économique. Porter $^{2}$ et Florida ${ }^{3}$ en sont peut-être les prosélytes les mieux connus, mais leur message est maintenant diffusé et appliqué du Michigan ${ }^{4}$ à la Finlande 5 , en passant par le sud de l'Angleterre ${ }^{6}$ et la Scandinavie ${ }^{7}$.

Le Québec n'est pas exempt de ce phénomène : la Cité du multimédia, les centres de la nouvelle économie et autres politiques visant à créer des grappes localisées font tous appel à l'idée que le regroupement physique d'intervenants dans un secteur donné entraînera des synergies, une dynamique locale et, par la suite, un avantage compétitif durable pour l'économie.

Cet article se propose, à partir d'une revue de la littérature, de répondre à plusieurs interrogations que suscitent ces politiques et ces écrits :

1) Ces idées sont-elles vraiment nouvelles ?

2) Le lien entre innovation et territoire est-il établi ?

3) Si les idées ne sont pas nouvelles et si le lien territoire - innovation reste à démontrer, a-t-on intérêt à miser dessus pour le développement des régions québécoises?
Nous abordons donc, en premier, la question de l'innovation. La deuxième partie explorera le lien entre innovation et territoire par le biais des idées sur les milieux innovateurs. La conclusion résumera les arguments sur l'innovation et le territoire et fera ressortir les conséquences en matière de politiques publiques (celles de développement régional) de ce questionnement.

\section{L'innovation}

Le concept d'innovation est vaste et ses emplois se rattachent à des sens variés, pouvant aller de l'innovation minimale opérée par un entrepreneur sur un chantier ${ }^{8}$ jusqu'à une innovation institutionnelle majeure comme l'avènement de la démocratie ${ }^{9}$. Mais dans un contexte de développement économique, et dans le discours actuel, la notion d'innovation est souvent cantonnée à son aspect technologique, et le lien entre innovation et dépenses en recherche et développement, ou entre innovation et brevets, est souvent mis en avant.

Or, cette vision étroite de l'innovation ne cadre pas avec la vaste littérature qui, depuis plus de cent ans, met l'accent sur le lien entre innovation et développement. En effet, selon plusieurs auteurs, l'innovation est un facteur central de l'évolution de la civilisation ${ }^{10}$.

Toynbee, en écrivant sur la révolution industrielle, la décrit comme étant à la fois technique et institutionnelle. Il ne sépare pas la régulation (syndicats, commerce, gouvernement) de la production (technologie, machines, finance) et voit le tout comme formant un système. Schumpeter, lorsqu'il décrit les conditions nécessaires au succès de l'entrepreneur (vecteur de l'innovation), fait ressortir l'importance de ce 
phénomène : « reaction of the social environment against one who wished to do something new »; l'innovation est donc rendue possible en partie grâce à un environnement social propice. Mumford introduit son livre par un chapitre sur la «Cultural preparation » : il y recense en détail les éléments socioculturels qui, selon lui, entraînent l'innovation technologique. Dans la mesure où une civilisation est urbanisée, l'exploitation des innovations a tendance à se faire en milieu urbain, quelle que soit leur origine ${ }^{11}$. Arthur Lewis, économiste spécialisé dans le développement économique, divise son livre sur la théorie de la croissance économique en sept chapitres : le deuxième s'intitule «Institutions économiques », parmi lesquelles il inclut à la fois les institutions formelles et informelles, le troisième chapitre s'intitule «Savoir» et le septième, «Gouvernement ». Dans le troisième chapitre, il analyse l'importance des attitudes envers l'innovation et envers l'application pratique de nouvelles idées, et dans le septième, il souligne l'importance des institutions gouvernementales. Solow, dans son célèbre article sur la théorie néoclassique de la croissance, identifie, à partir d'un raisonnement économétrique, un « résidu » de croissance inexplicable par les facteurs traditionnels (croissance de population, de capital et de main-d'œuvre). Ce résidu constitue la mise en évidence mathématique de l'effet des changements institutionnels et techniques identifiés précédemment de manière empirique. Drucker souligne, pour un public de généralistes, l'avènement de la société du savoir, la globalisation, l'affaiblissement des gouvernements nationaux face à ces tendances et d'une nouvelle économie ${ }^{12}$. Finalement, Landes, dans sa conclusion, émet cette idée : «ideo-logy is only one of many non-economic factors autonomous in origin but closely connected both as cause and effect in the Industrial revolution ».

L'ensemble de ces auteurs, qui ont réfléchi et publié au cours du siècle précédent - et qui l'ont fait avant l'avènement de la crise des années 1970, d'Internet, des ordinateurs portatifs, des téléphones cellulaires, du télécopieur -, s'accordent pour mettre en évidence l'innovation comme facteur clé de la croissance. De plus, ils sont en accord sur le fait que les institutions, idéologies et cultures sont à la base des innovations technologiques et que le lien entre technologie et culture est ambigu. Si une direction causale devait être identifiée, elle irait, sur la base des auteurs cités, de la culture vers l'innovation technologique, et non en sens inverse. Cependant, ils ne font pas de lien entre innovation et territoire. Lorsqu'ils parlent de «pays », ils font davantage référence à des entités avec des cultures et des institutions homogènes qu'à des terrires.

L'ensemble des auteurs étudiés, qui ont réfléchi et publié au cours du siècle précédent, s'accordent pour mettre en évidence l'innovation comme facteur clé de la croissance. De plus, ils sont en accord sur le fait que les institutions, idéologies et cultures sont à la base des innovations technologiques et que le lien entre technologie et culture est ambigu. Cependant, ils ne font pas le lien entre innovation et territoire.

Une autre remarque que nous pouvons faire concernant cette recension rapide est qu'elle ne nous permet pas de saisir avec exactitude ce qu'est une innovation. L'ensemble des auteurs s'accordent sur son importance, beaucoup d'exemples très pertinents en sont donnés, mais aucune définition n'en ressort. Or, s'il est question d'interventions politiques, une définition opérationnelle est nécessaire. Aucune définition du concept n'existe cependant.

À notre avis, ceci est tout à fait normal car l'innovation est un terme relatif (on innove par rapport à quelque chose) et qualitatif (il existe divers degrés d'innovation, et ces degrés dépendent en partie de jugements individuels). Il est vrai que certaines institutions, comme l'OCDE (Organisation de coopération et de développement économique), ont tenté de définir l'innovation, mais ces institutions parviennent à une définition au prix de grandes restrictions : le manuel d'Oslo $^{13}$ se cantonne à la définition des innovations scientifiques et technologiques au sein d'entreprises. Dans le cadre restreint de cette définition, la standardisation proposée par l'OCDE est fort utile (et débattue), mais elle ne résout pas les problèmes mentionnés ci-dessus (relativité et subjectivité), et elle ne tient pas compte du cadre beaucoup plus large dans lequel l'innovation doit être comprise. La littérature recensée ci-dessus nous montre que le concept d'innovation est un concept utile, même central, mais flou ; 
toute définition trop précise en laissera de côté certaines facettes.

Une autre remarque importante ressort de cette recension : le lien entre innovation et développement économique n'est pas nouveau. Or, si le lien est reconnu depuis longtemps et si, jusqu'à présent, les politiques de développement régionales n'en ont pas profité, pourquoi tant d'emphase aujourd'hui sur ces anciens concepts ?

\section{Les milieux innovateurs}

Pour répondre partiellement à cette question, nous nous tournerons maintenant vers la notion de milieu innovateur. C'est en effet par le biais de milieux liés au territoire que le lien entre innovation et développement économique est censé s'effectuer.

Il n'est pas de notre intention de faire ici une recension complète de la littérature récente sur ce phénomène, ni même d'en établir une définition trop détaillée. Les écrits de Braczyk et autres ${ }^{14}$, Malecki et Oinas ${ }^{15}$, Brown et Duguid ${ }^{16}$, pour n'en nommer que quelquesuns, le font très bien. Mais, à partir de ces écrits, il est relativement aisé de dresser un portrait sommaire de ce qui est entendu par ce terme ainsi que d'identifier certaines raisons qui motivent sa résurgence à l'heure actuelle. En gros, un milieu innovateur est un ensemble d'acteurs économiques et institutionnels liés par une culture commune et qui, par un jeu de collaboration et de compétition, parviendraient à maintenir un avantage compétitif ${ }^{17}$. Cet avantage serait maintenu par le biais de l'innovation constante en matière de procédés et de produits finaux que générerait la synergie du milieu. Cette notion est souvent rattachée au territoire, car la proximité physique favoriserait ces synergies : le milieu innovateur devient alors une zone géographique dans laquelle les interactions décrites ci-dessus auraient lieu ${ }^{18}$.

Mais ici aussi, il est important de bien se rendre compte que les concepts n'ont pas grand-chose de nouveau : la plus grande nouveauté, depuis une dizaine d'années, est l'intérêt qu'y portent les chercheurs et les décideurs. En effet, le « milieu », dans un contexte de développement économique, est un phénomène ancien et non une découverte nouvelle liée à la «nouvelle économie $»^{19}$. La plupart des chercheurs actuels reconnaissent que Marshall ${ }^{20}$, lorsqu'il décrivait les districts industriels de l'ère victorienne, décrivait en somme ce que l'on appellerait aujourd'hui un milieu innovateur. Brown et Duguid font clairement le lien entre les notions véhiculées aujourd'hui (importance de la proximité physique et culturelle, mobilité de la maind'œuvre, transmission du savoir-faire tacite, coopération compétitive entre intervenants, cadre institutionpropice) et celles décrites par Marshall.

Un milieu innovateur est un ensemble d'acteurs économiques et institutionnels liés par une culture commune et qui, par un jeu de collaboration et de compétition, parviendraient à maintenir un avantage compétitif. Cet avantage serait maintenu par le biais de l'innovation constante en matière de procédés et de produits finaux que générerait la synergie du milieu. Cette notion est souvent rattachée au territoire, car la proximité physique favoriserait ces synergies : le milieu innovateur devient alors une zone géographique dans laquelle les interactions décrites ci-dessus auraient lieu.

Cependant, il existe une distinction centrale entre le monde dans lequel vivait Marshall et celui dans lequel nous vivons aujourd'hui : à son époque, la mobilité des individus et le maintien de réseaux à distance étaient difficiles. Le transport de marchandises surtout des marchandises intermédiaires - se faisait plus difficilement et donc, pour certains secteurs et dans certaines filières, la proximité géographique devait jouer un rôle important en ce qui a trait au réseautage. L'argument central de cet article est qu'aujourd'hui, le territoire entre principalement en jeu comme noeud dans une série de réseaux, comme lieu de haute accessibilité aux marchés, à la main-d'œuvre et aux infrastructures (ces dernières permettant justement l'accès et le maintien des réseaux à distance). Par contre, le réseautage local ne prime plus nécessairement sur le réseautage à d'autres échelles, et la notion de milieu innovateur territorialisé n'est donc pas généralisable.

Marshall lui-même employait un vocabulaire révéla- 
teur : il a en effet souligné l'importance des «mystères » d'une filière. Il entendait par là l'ensemble des connaissances tacites, des savoir-faire et des institutions informelles qui liaient les entreprises œuvrant dans un territoire donné. Or, à l'origine, ce terme ne comportait aucune connotation géographique. Les «mystères » étaient un ensemble de conventions secrètes qui permettaient aux membres itinérants d'une guilde (par exemple celle des francs-maçons) de se reconnaître ${ }^{21}$. À l'époque médiévale, où la vérification des compétences et des qualifications de travailleurs itinérants était ardue, il était de toute première importance de pouvoir reconnaître la main-d'œuvre qualifiée.

Ceci nous apprend deux choses : d'une part, à l'époque médiévale, l'importance des connaissances tacites était bien reconnue, même si le concept n'était pas articulé de la même façon qu'il l'est aujourd'hui. La méthode de formation privilégiée de l'époque était l'apprentissage ${ }^{22}$ - la méthode par excellence de transmission des connaissances tacites -, et les «mystères » servaient à reconnaître ceux qui avaient suivi cette formation. La deuxième chose que cela nous apprend est que la main-d'œuvre qualifiée était mobile, rendant nécessaire ce système secret d'identification. Autrement dit, les connaissances tacites n'étaient pas figées (même à une époque qui n'est pas connue pour sa grande mobilité). Le «milieu » des artisans spécia-lisés n'était pas lié au territoire ; c'est iustement la raison pour laquelle il leur était nécessaire développer ces « mystères ».

L'argument central de cet article est qu'aujourd'hui, le territoire entre principalement en jeu comme noeud dans une série de réseaux, comme lieu de haute accessibilité aux marchés, à la main-d'œuvre et aux infrastructures. Par contre, le réseautage local ne prime plus nécessairement sur le réseautage à d'autres échelles, et la notion de milieu innovateur territorialisé n'est donc pas généralisable.

De toute évidence, certaines filières économiques étaient et restent liées au territoire. Il serait difficile d'avoir une filière de la pêche en Abitibi, et la filière de la coutellerie à Thiers en France a sans doute bénéficié de la présence du minerai de fer ainsi que de la rivière $^{23}$. Mais ces filières se sont développées à ces endroits précis non pas, en premier lieu, à cause de la présence d'autres acteurs économiques, mais bien à cause de la présence de caractéristiques géographiques propices. Les innovations et la spécialisation du travail sont venues par la suite. La notion de « milieu innovateur », surtout si elle est censée servir au développement régional, voudrait que la simple colocalisation d'acteurs économiques et d'institutions propices mène, par l'innovation, à la croissance économique. Même à la lumière des écrits de Porter sur les avantages compétitifs, cela peut paraître irréaliste, ne serait-ce qu'à cause de l'importance de plus en plus réduite des ressources premières comme élément d'avantage comparatif $^{24}$.

Cela dit, on prétend souvent aujourd'hui que le fonctionnement de l'économie a profondément changé. La nouvelle économie ${ }^{25}$ fait en sorte que les leçons tirées de l'histoire seraient au mieux anecdotiques, au pire trompeuses. L'avènement des nouvelles technologies de production et d'information aurait profondément changé la manière de fonctionner de l'économie, et notamment son rapport à l'espace.

Or, sans nier que les choses évoluent, il n'est pas certain que les différences avec les exemples historiques soient aussi profondes qu'on le prétend, que ce soit en matière d'introduction de nouvelles technologies de communication ${ }^{26}$ ou de l'annonce de nouvelles ères économiques $^{27}$. Peu de chercheurs et d'acteurs prétendent qu'un avantage compétitif peut être créé ex nihilo: les avantages compétitifs régionaux sont bâtis sur des forces régionales ${ }^{28}$. À Thiers, c'est un avantage compétitif qui a été créé sur la base d'un avantage comparatif existant. C'est le minerai de fer (avantage comparatif disponible à plusieurs endroits en France) qui a rendu possible la création du milieu (avantage compétitif unique); mais ce milieu ne serait pas apparu, minerai ou pas, sans la présence de main-d'œuvre, d'une situation centrale (l'accès aux marchés), et de la chance, le «first mover advantage » (qui est dû, selon la légende, à l'importation par des soldats de retour de croisades, du secret de la fabrication, qu'eux-mêmes avaient recueilli en Orient).

Autrement dit, il nous est impossible de préciser avec exactitude les raisons pour lesquelles la filière de la coutellerie s'est installée précisément à Thiers. Mais 
une fois la filière établie, il nous est plus facile de comprendre que la spécialisation du travail et la croissance de secteurs connexes (bijoutiers, négociants, etc.) aient mené à ce que l'on pourrait appeler un milieu. Scott ${ }^{29}$ en arrive à des conclusions très semblables quand il décrit le développement du secteur de "ronautique dans le comté d'Orange à Los Angeles.

Le phénomène de milieu est une réalité ancienne, même si son articulation en termes scientifiques est plus récente. Ce n'est pas, au fond, la réalité des phénomènes économiques locaux qui évolue, mais la nature des avantages compétitifs et comparatifs. Le discours sur les milieux innovateurs ne semble être qu'une nouvelle conceptualisation de ces anciens phénomènes.

Face à ces dynamiques, une région qui n'a pas d'avantage particulier dans la nouvelle économie peut-elle devenir un milieu innovateur par le biais d'interventions politiques ? Cela paraît peu probable. Mais une région déjà avantagée pourra sans doute renforcer cet avantage en s'inspirant des idées sur les milieux innovateurs. Quels sont donc les avantages régionaux dans la nouvelle économie ? Ce sont de moins en moins des avantages comme la présence d'un minerai, et de plus en plus ceux tournant autour de la facilité de communication, de l'accès aux marchés et de l'accès à la main-d'œuvre. C'est dans des régions présentant de telles caractéristiques que pourront, éventuellement, se développer des milieux donc dans des territoires urbains et métropolitains.

En somme, le phénomène de milieu est une réalité ancienne, même si son articulation en termes scientifiques est plus récente. Ce n'est pas, au fond, la réalité des phénomènes économiques locaux qui évolue, mais la nature des avantages compétitifs et comparatifs. Le discours sur les milieux innovateurs ne semble être qu'une nouvelle conceptualisation de ces anciens phénomènes. Or, si les phénomènes évoluent peu, nous devons nous poser la question à savoir si de nouvelles approches au développement qui reposent sur ceux-ci sont nécessaires pour répon-
Quels sont donc les avantages régionaux dans la nouvelle économie ? Ce sont de moins en moins des avantages comme la présence d'un minerai, et de plus en plus ceux tournant autour de la facilité de communication, de l'accès aux marchés et de l'accès à la main-d'œuvre.

dre à des processus plutôt statiques ou en évolution lente.

\section{Conclusion}

\section{Territoires et innovation : existe-t-il un lien?}

Le fait que certains secteurs économiques se soient concentrés sur certains territoires et qu'une division du travail s'y soit développée n'a rien de nouveau. Ce phénomène est en évidence, au moins depuis l'époque médiévale. Mais, même à cette époque, où les communications étaient beaucoup moins efficaces, le lien entre milieu et territoire n'était pas absolu : les guildes d'artisans itinérants, les liens économiques et intellectuels qui suivaient les grands axes commerciaux internationaux ${ }^{30}$ créaient aussi des milieux non localisés dans lesquels s'échangeaient savoir-faire, intrants et extrants et main-d'œuvre spécialisée. La plus grande nouveauté réside donc dans le discours et la conceptualisation de ces phénomènes, et non dans les phénomènes eux-mêmes.

Très récemment, plusieurs chercheurs ont été interpellés par ce discours portant sur le lien entre milieux innovateurs et territoire ; ils ont remis en question cet attachement territorial par le biais d'études empiriques

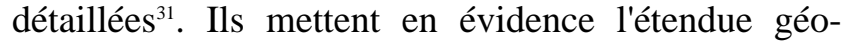
graphique des réseaux, réseaux qui ne sont pas limités par la proximité physique. Les NTIC, qui facilitent ce réseautage à distance, ont un effet géographique paradoxal : elles concentrent l'activité économique autour des grands centres métropolitains ${ }^{32}$ tout en facilitant l'identification d'informations et la gestion de réseaux aspatiaux $^{33}$. D'ailleurs, certains auteurs parlent de réseaux de villes, chaque ville se spécialisant dans telle ou telle activité et dépendant des autres. Les réseaux d'entreprises (comme, par exemple, les 
eaux dans le domaine de la finance) se calqueraient is sur le réseau de villes que sur le milieu local ${ }^{34}$.

L'existence des grappes et autres concentrations géographiques d'activités serait plus aisément explicable par la présence d'infrastructures permettant l'accès à ces réseaux de maind'œuvre qualifiée, d'employés et d'employeurs nombreux et variés, enfin, par les externalités positives de l'agglomération, que par les effets de milieu. La territorialisation de l'innovation et les effets de milieu, bien qu'ils ne soient pas à exclure, ne sont que des explications marginales de ces concentrations géographiques et se confondent donc avec les attributs d'une métropole.

L'existence des grappes et autres concentrations géographiques d'activités serait donc plus aisément explicable par la présence d'infrastructures permettant l'accès à ces réseaux de main-d'œuvre qualifiée, d'employés et d'employeurs nombreux et variés, enfin, par les externalités positives de l'agglomération, que par les effets de milieu. La territorialisation de l'innovation et les effets de milieu, bien qu'ils ne soient pas à exclure, ne sont que des explications marginales de ces concentrations géographiques ${ }^{35}$ et se confondent donc avec les attributs d'une métropole.

\section{Politiques de développement}

basées sur l'innovation et le territoire ?

La discussion sur la pertinence de politiques de développement régionales basées sur le lien présumé entre innovation et territoire ne pouvait avoir lieu qu'en fin d'article, car il fallait d'abord établir la fragilité de nos définitions opérationnelles de l'innovation ainsi que la pérennité de l'idée de milieu.

Si nous ne savons pas exactement ce qu'est l'innovation et si les milieux existent depuis longtemps et ne sont pas nécessairement liés au territoire, alors il nous est impossible de proposer une approche générale au développement sur la base de ces concepts.
L'innovation, dans son sens le plus large, a lieu partout. L'usine à Baie-Comeau qui invente une nouvelle manière de façonner le bois, les chercheurs en Gaspésie qui exploitent des déchets animaux, les ingénieurs en Abitibi qui explorent de nouvelles techniques minières, tous innovent. La plupart le font en se reposant en partie sur leur propre imagination et connaissance, en partie sur le milieu immédiat dans lequel ils se trouvent, et en partie sur les maintes interactions qu'ils entretiennent, à travers Internet, les voyages, le contact avec les clients, avec le reste du monde. Prétendre que leurs innovations sont issues du « milieu local » revient à minimiser leur apport personnel et celui de l'extérieur, apports qui sont parfois plus importants.

Si nous ne savons pas exactement ce qu'est l'innovation et si les milieux existent depuis longtemps et ne sont pas nécessairement liés au territoire, alors il nous est impossible de proposer une approche générale au développement sur la base de ces concepts.

La notion de « milieu » véhiculée dans la littérature, et reprise dans certaines politiques publiques, correspond à ce que l'on peut trouver en métropole ${ }^{36}$. Mais même en métropole, les contacts avec le monde extérieur et le génie individuel restent centraux pour l'innovation. Cependant, il est évident que la présence de recherche institutionnalisée, de division accrue du travail et d'une masse plus grande d'acteurs économiques sont aussi des facteurs de première importance. Tout ceci est effectivement réuni en un endroit assez restreint menant à la conclusion - qui à mon avis n'a pas encore été démontrée ${ }^{37}$ - qu'il existe un lien entre territoire et innovation.

Mais ce lien pourrait n'être qu'apparent. Ce n'est pas parce que certaines métropoles accueillent plus d'interactions productives et innovantes que d'autres que le territoire métropolitain est la cause de l'innovation. Cela reviendrait à prétendre que le bol dans lequel on mélange la pâte à crêpes est la «cause » des crêpes. Ce n'est évidemment pas le cas : ce sont bien les interactions et les individus qui sont la «cause » de l'innovation en métropole, et les interactions entre les ingrédients qui «causent» la pâte dans le bol. À la dif- 
férence de la pâte à crêpes, qui a besoin du bol pour qu'aient lieu les interactions entre les aliments, les interactions entre individus ont de moins en moins besoin de s'effectuer à proximité. La proximité sert à l'accès aux marchés (soit ils sont locaux, soit ils sont accessibles par les réseaux de transport), à la maind'œuvre et aux infrastructures, mais n'est pas nécessaire pour l'innovation. Le face-à-face, qui demeure très important, s'effectue lors de colloques, de foires et voyages.

Ce n'est pas parce que certaines métropoles accueillent plus d'interactions productives et innovantes que d'autres que le territoire métropolitain est la cause de l'innovation. Cela reviendrait à prétendre que le bol dans lequel on mélange la pâte à crêpes est la « cause » des crêpes. Ce n'est évidemment pas le cas : ce sont bien les interactions et les individus qui sont la « cause » de l'innovation en métropole, et les interactions entre les ingrédients qui « causent » la pâte dans le bol.

Ceci se remarque justement en région. Les entrepreneurs y sont très innovateurs et mettent en pratique leurs innovations. Mais ces innovations, souvent, sont peu ou aucunement liées au territoire immédiat dans lequel évoluent les innovateurs. Cependant, s'ils veulent « développer» ou «commercialiser» leur produit, il leur est souvent nécessaire d'aller vers la métropole. Là se trouvent la finance, la main-d'œuvre, les laboratoires, les aéroports, les clients permettant la pleine exploitation de leur innovation. Encore une fois, il serait fallacieux d'examiner le résultat final et de prétendre que ces innovations émanent de la métropole alors que les premières idées et applications ont jailli ailleurs. Il suffit de se souvenir que Bombardier a commencé ses innovations à Valcourt et non à Montréal pour comprendre comment une telle erreur est facile à faire.

Ces processus de métropolisation sont peut-être inéluctables. Les avantages d'une métropole en matière d'accès à la main-d'œuvre et à la clientèle, avantages de tout premier ordre pour le développement et la commercialisation d'une innovation, ne sont pas facilement reproductibles en région. Mais il ne faut pas confondre l'innovation en soi, qui peut avoir lieu n'importe où et qui est issue de la confluence de plusieurs courants qui ne sont pas nécessairement liés au territoire, et l'exploitation de l'innovation qui elle, peut effectivement mieux se développer dans certains ritoires que dans d'autres.

Il ne faut pas confondre l'innovation en soi, qui peut avoir lieu n'importe où et qui est issue de la confluence de plusieurs courants qui ne sont pas nécessairement liés au territoire, et l'exploitation de l'innovation qui elle, peut effectivement mieux se développer dans certains territoires que dans d'autres.

En somme, les politiques de développement régionales visant à stimuler les milieux innovateurs reposent, pour l'instant, sur des bases théoriques incertaines. Le lien entre innovation et territoire reste à démontrer. Ce lien peut paraître évident pour les métropoles, mais il existe des explications alternatives qui doivent être envisagées avant que l'on puisse affirmer avec confiance qu'un lien existe entre le territoire d'une métropole et l'innovation. Même dans les grandes villes, les idées et les réseaux s'étendent bien au-delà des frontières urbaines, et le territoire ne joue peutêtre que le rôle d'un bassin (ou d'un bol ?) de maind'œuvre, d'infrastructures et de marchés.

En ce qui concerne les régions non métropolitaines, il est certain que des innovations y ont lieu de manière continue. Mais ces régions ne semblent pas en profiter en matière d'emplois et de développement car elles ne présentent pas les mêmes avantages que les métropoles pour exploiter ces innovations. Donc, le fait d'encourager l'innovation en région, sans être négatif ou nuisible, n'aura sans doute pas les effets régionaux escomptés en matière de développement et d'emploi.

Le but de cet article n'est pas de prétendre que les politiques de développement régionales construites autour de la notion de milieu innovateur n'auront nécessairement aucun effet. Le but st plutôt d'attirer l'attention 
sur certaines questions fondamentales qui ne semblent pas encore avoir été résolues et d'alimenter le débat autour de ces questions.

\section{Notes et références}

1 Certains éléments de cet article ont été présentés à la conférence de l'ASDEQ, en mai 2001. Je remercie les participants à ce colloque, ainsi que Laurent Terral, pour les discussions intéressantes qui ont permis de faire avancer ces réflexions.

2 PORTER, M. (1990). The Competitive Advantage of Nations, New York, Free Press.

3 FLORIDA, R. (1995). «Toward the learning Region", Futures, vol. 27, no 5, p. 527-536.

4 CSLR (Centre for Sustained Learning Regions) (2001). $\mathrm{http} / / / \mathrm{mtip}$. net/regions/centerstaff.htm.

5 LUT (Lappeerannan teknillinen korkeakouly) (2001). Learning Wood Region, http://developmentcentre.lut.fi/lwr/lear_wood.htm.

6 SEEDA (2001). Building a World Class Region: an Economic Strategy for the South East of England, Guildford, South East England Development Agency.

7 MASKELL, A. and G. TÖRNQUIST (2000). Building $a$ Cross-Border learning Region, Copenhagen, Copenhagen School Business Press.

8 HOLBROOKE, A., T. PADMORE and L. HUGHES (2000). «Innovation in Enterprises in a Non-Metropolitan Area: Quantitative and Qualitative Perspective », in Holbrooke, A. et D. Wolfe (éd.), Innovations, Institutions and Territory: Regional Innovation Systems in Canada, Montreal and Kingston, McGill-Queen's University Press.

9 OLSON, M. (2000). Power and Prosperity, Basic Books, New York.

10 TOYNBEE, A. (1884-1962), The Industrial Revolution, Boston, Beacon Press; SCHUMPETER, J. (1936). The Theory of Economic Development, Cambridge, Harvard University Press (first German edition published in 1911) ; MUMFORD, L. (1934). Technics and Civilzation, New York, Harcourt Brace \& World Inc. ; LEWIS, A. (1955). The Theory of Economic Growth, London, George Allen Unwin ; SOLOW, R. (1956). "A contribution to the Theory of Economic Growth », Quarterly Journal of Economics, 70, p. 65-94 ; DRUCKER, P. (1968). The Age of Discontinuity, New York, Harper \& Row ; LANDES, D. (1969). The Unbound Prometheus: Technological Change and Industrial Development in Western Europe from 1750 to the Present, Cambridge, Cambridge University Press.

11 MUMFORD, L. (1961). Cities in History, New York, Harcourt Brace Jovanovitch. Nous reviendrons plus loin sur la distinction entre l'innovation et sa première mise en pratique, et entre l'exploitation et la commercialisation de l'innovation.

12 La «nouvelle économie » des années 1960 était l'économie keynésienne. Drucker (1968) propose d'aller au-delà des schémas économiques traditionnels de l'époque pour y intégrer les industries de service, une théorie de l'innovation et une compréhension de l'interaction systémique entre micro et macro-économie.

13 OCDE (1997). Oslo Manual (Second edition), Paris, OCDE.

14 BRACZYK, H. J., P. COOKE and M. HEIDENREICH (éd.) (1998). Regional Innovation Systems: the Role of Governance in a Globalized World, London, UCL Press.

15 MALECKI, E. and P. OINAS (éd.) (1999). Making Connections: Technological learning and Regional Economic Change, Brookfield, Ashgate.

16 BROWN, J. and P. DUGUID (2000). «Mysteries of the Region: Knowledge in Silicon Valley », in Millar, W., C. M. Lee, M. Hancock and H. Rowen (éd.), The Silicon Valley Edge: a Habitat for Innovation and Entrepreneurship, Stanford, Stanford University Press.

17 PORTER, M. (1990). Op. cit., note 2.

18 MAILLAT, D. (1992). «Milieux et dynamique territoriale de l'innovation », Revue canadienne des sciences régionales, vol. $15, \mathrm{n}^{\circ} 2$, p. 199-218.

19 PERRIN, J.-C. (1992). «Pour une révision de la science régionale : l'approche par les milieux », Revue canadienne des sciences régionales, vol. $15, \mathrm{n}^{\circ} 2$, p. 155-198.

20 MARSHALL, A. (1890). Principles of Economics: An Introductory Volume, London, Macmillan.

21 En fait, le terme « mystère » correspond à la fois aux connaissances tacites elles-mêmes, c'est-à-dire au savoir-faire et à la manière de reconnaître rapidement les possesseurs de ce savoir-faire.

22 GIMPEL, J. (1975). La Révolution industrielle du Moyen Âge, Paris, Éditions du Seuil.

23 THIERS (2001). Site officiel de la coutellerie : http://www.coutellerie-thiers.com/historique.htm.

$24 \mathrm{La}$ principale distinction entre «avantage compétitif » et «avantage comparatif » est la suivante : un avantage compétitif est un avantage qui se crée, qui doit être maintenu et qui peut s'effriter. La présence dans un lieu donné d'un système éducatif particulièrement performant en est un exemple. Un avantage comparatif a un caractère plus absolu et ne dépend pas des institutions ou des politiques locales. La présence d'un minerai en est un exemple. Il est clair que l'un et l'autre avantage ne demeurent un "avantage » que s'il existe une demande ; ni l'un ni l'autre n'est pérenne. En ce moment, il semblerait que les avantages comparatifs liés à la géographie s'effritent. Selon Porter (1990), certaines régions parviennent à se créer des avantages compétitifs : la question est de savoir si ces avantages ne recoupent pas, en fait, les avantages liés à l'agglomération d'activités économiques, en particulier l'accès à la main-d'œuvre, aux marchés et aux infrastructures.

25 CSLS (Centre for the Study of Living Standards) (2000). Trend 
Productivity and the New Economy, Ottawa, CSLS ; OCDE (2000). A New Economy? The Changing Role of Innovation and Information Technology in Growth, Paris, OCDE.

26 GORDON, R. (2000). Does the «New Economy» Measure up to the Great Inventions of the Past?, NBER working paper 7833, Cambridge, NBER.

27 SHILLER, R. (2001). Irrational Exuberance, New York, Broadway Books.

28 PORTER, M. (1990). Op. cit., note 2.

29 SCOTT, A. (1988). New Industrial Spaces, Londres, Pion.

30 BRAUDEL, F. (1979). Les Jeux de l'Échange, Paris, Armand Collin.

31 SUAREZ-VILLA, L. and W. WALROD (1997). « Operational Strategy, R\&D and Intrametropolitan Clustering in a Polycentric Structure: The Advanced Electronics Industries of the Los Angeles Basin », Urban Studies, vol. 34, no 9, p. 1343-1380 ; SIMMIE, J. (1998). «Reasons for the Development of Islands of Innovation: Evidence from Hertfordshire », Urban Studies, vol. 35, no 8, p. 1261-1289 ; ECHEVERRI-CARROLL, E. and W. BRENNAN (1999). «Are Innovation Networks Bounded by Proximity?», in M. Fischer, L. Suarez-Villa and M. Steiner (éd.), Innovation, Networks and Localities, New York, Springer-Verlag; SIVITANIDOU, R. (1999). «The Location of Knowledgebased Activities: the Case of Computer Software in Innovation, Networks and Localities », in M. Fischer, L. Suarez-Villa and M. Steiner (éd.), Innovation Networks and Localities, New York, Springer-Verlag.

32 GRAHAM, S. and S. MARVIN (1996). Telecommunications and the City, London, Routledge.
33 CASTELLS, M. (1996). The Rise of the Network Society, London, Blackwell.

34 SASSEN, S. (1996). "The Global City», Urban Theory, S. Fainstein and S. Campbell (éd.), Oxford, Blackwell ; BATTEN, D. (1995). "Network Cities: Creative Urban Agglomerations for the $21^{\text {st }}$ Century », Urban Studies, vol. 32, p. $313-327$.

35 SIMMIE, J. (1998). Op. cit., note 31 ; SIVITANIDOU, R. (1999). Op. cit., note 31.

36 CRÉVOISIER, O. (1999). «Innovation and the City », in E. Malecki and P. Oinas (éd.), Making Connections: Technological Learning and Regional Economic Change, Brookfield, Ashgate.

37 Il a été démontré sans équivoque par de nombreux chercheurs qu'à l'intérieur de certains territoires, il semble exister un réseautage important qui pourrait mener à l'innovation. Ce qui n'a pas été démontré est que ce réseautage interne au territoire est moins significatif que le réseautage externe au territoire, ni que ce réseautage interne peut être distingué des contacts normaux qu'entretiennent des acteurs économiques proches les uns des autres. En effet, ce n'est pas parce que $90 \%$ des interactions économiques se font dans un rayon de trois kilomètres autour de l'entreprise (achat de fournitures, soustraitance banale, services financiers de routine, consultation technique ordinaire) que les interactions les plus importantes mais les moins fréquentes (premier appel à l'épargne publique, collaborations spécialisées, consultations pointues, idée géniale lors d'une conférence à l'étranger) ne se font pas à une toute autre échelle géographique. Il y a donc une question d'échelle, une question de biais des études vers les réseaux locaux, mais aussi une question sur la nature précise des réseaux et des interactions. Sans réponse à ces questions, il me semble que le lien présumé entre territoire et innovation (en 